\title{
La mobilité transnationale comme ressource : le cas des migrants de l'Europe de l'Est
}

\section{Mirjana Morokvasic-Muller}

\section{(2) OpenEdition \\ 1 Journals}

\section{Édition électronique}

URL : http://journals.openedition.org/conflits/263

DOI : $10.4000 /$ conflits.263

ISSN : $1777-5345$

Éditeur :

CCLS - Centre d'études sur les conflits lilberté et sécurité, L'Harmattan

Édition imprimée

Date de publication : 15 mai 1999

ISSN : 1157-996X

Référence électronique

Mirjana Morokvasic-Muller, «La mobilité transnationale comme ressource : le cas des migrants de I'Europe de l'Est », Cultures \& Conflits [En ligne], 33-34 | printemps-été 1999, mis en ligne le 16 mars 2006, consulté le 30 mars 2021. URL : http://journals.openedition.org/conflits/263 ; DOI : https:// doi.org/10.4000/conflits.263

Ce document a été généré automatiquement le 30 mars 2021.

Creative Commons License 


\title{
La mobilité transnationale comme ressource : le cas des migrants de l'Europe de l'Est
}

\author{
Mirjana Morokvasic-Muller
}

1 Depuis le début des années 1990 le paysage migratoire a changé : les flux se sont diversifiés, les lieux d'origine et de destination se sont multipliés, imbriqués les uns dans les autres par une nouvelle dynamique où la mobilité joue un grand rôle dans les stratégies des acteurs ${ }^{1}$. De toutes les dimensions qui caractérisent le nouveau paysage migratoire européen depuis la fin des années 80, l'une des plus importantes est la possibilité qu'ont la plupart des ressortissants de l'Europe centrale et orientale non seulement de partir mais aussi de revenir dans leur pays d'origine. C'est la réunion de ces deux conditions qui donne un sens nouveau au potentiel migratoire d'une population certes prête à partir, mais pas forcément à émigrer : la confusion entre le potentiel et la " pression migratoire » fut longtemps entretenue à l'Ouest comme à l'Est de l'Europe au moyen de scénarios-catastrophe ${ }^{2}$ avec l'idée héritée de la littérature des années 80 que "tôt ou tard les migrants finiraient par s'installer dans le pays d'arrivée " ${ }^{3}$. Dans ce texte il sera question des migrations qui contribuent, entre autres, à alléger la pression migratoire, notamment des mouvements circulatoires ou pendulaires, l'accent étant mis sur les stratégies migratoires des acteurs. Compte tenu de la nature même de leur migration, les problématiques longtemps centrales dans la recherche en ce domaine, à savoir l'intégration ou le retour, s'avèrent d'une pertinence marginale. La « libre circulation " ${ }^{4}$ entre l'Est et l'Ouest européen se développe dans un espace « frontalier » de plus en plus vaste et dans un contexte où l'Europe de l'Ouest est quasiment fermée à l'immigration : d'une part, elle n'admet à titre permanent qu'un nombre limité de personnes dont le profil varie d'un pays à l'autre, et, d'autre part, elle n'entrouvre ses portes que pour la migration temporaire de travail et de moins en moins pour les demandeurs d'asile. L'explosion de la mobilité transfrontalière et les mouvements de courte durée sous forme de navettes ${ }^{5}$ sont souvent considérés comme caractéristiques de l'Europe centrale et orientale ${ }^{6}$. Or, comme l'ont montré les travaux de Alain Tarrius, Michel Peraldi ${ }^{7}$ et d'autres recherches sur les réseaux transnationaux 
entre la France et le $\mathrm{Maghreb}^{8}$, ainsi que ceux d'anthropologues américains ${ }^{9}$, il ne s'agit pas d'une particularité de l'Europe de l'Est, mais d'un phénomène plus général lié à la mondialisation des échanges et des flux. A l'ouest européen et notamment en Allemagne, une multitude de statuts temporaires est censée gérer une partie des personnes destinées au marché du travail, que ce soit par le biais de nouveaux programmes pour "Gastarbeiter » et autres catégories de travailleurs embauchés pour des contrats de courte durée ${ }^{10}$, ou sous le couvert d'action humanitaire, la " protection temporaire " notamment ${ }^{11}$. Cependant, la grande majorité des gens «libérés " par les restructurations économiques à l'Est, par les conflits dits «ethniques » ou d'autres épisodes de la transition post-communiste, échappe à cette gestion ${ }^{12}$. Outre la courte durée des mouvements et leur caractère massif, la qualité et la diversité caractérisent cette population : toutes les couches sociales des sociétés de départ sont concernées. Il s'agit d'une population plutôt citadine, d'un niveau d'éducation et de qualification élevé (entre 11 et $23 \%$ de personnes hautement qualifiées). Par ailleurs, les femmes, pratiquement absentes des programmes de recrutements officiels ${ }^{13}$, sont parfois plus nombreuses que les hommes dans ces mouvements. La transition actuelle concerne des pays dans lesquels les femmes, qui avaient acquis certains droits et étaient économiquement actives, se retrouvent perdantes sur les plans économique, politique et social ${ }^{14}$. Compte tenu de la difficulté ou de l'impossibilité de trouver du travail dans leur pays d'origine, elles optent pour la migration. La circulation semble aussi être un élément important des migrations qu'on qualifie par ailleurs de migrations permanentes ou d'installation. On estime ainsi qu'un demi-million d'Aussiedler (Allemands ethniques) originaires de Pologne, ressortissants allemands naturalisés, continue d'avoir une double résidence et font des navettes entre la Pologne et l'Allemagne. Une enquête récente auprès des Juifs de l'ex-Union Soviétique en Allemagne (où plus de 60.000 ont été admis depuis 1990 comme réfugiés politiques) indique que l'émigration de deux tiers d'entre eux a été motivée par la proximité géographique de l'Allemagne et de la Russie et par la possibilité de faire des navettes pour des raisons commerciales ${ }^{15}$. La circulation marchande ou celle motivée par le travail constituent l'une des plus fréquentes manifestations des migrations dites de «transit ${ }^{16}$.

2 La libre circulation "est-européenne »: les marchands et travailleurs ambulants Il s'agit de la forme la plus répandue de cette libre circulation. Pour nombre de personnes à l'Est, la course précipitée vers l'économie de marché prend des allures de bazar. Pour éviter d'être marginalisés par des bouleversements socio-économiques de la société, ils se lancent dans une « économie liée aux voyages » (l'économie du bazar) et cherchent à améliorer leur situation en restant mobiles aussi longtemps qu'ils le peuvent. Ils déploient un dynamisme extraordinaire que l'embrigadement dans le système communiste n'a pas réussi à anéantir. Dans les villes et villages des pays en train de décoller ou cherchant à décoller économiquement après la chute du système communiste, on trouve des marchés plus ou moins organisés où des résidents locaux, mêlés à des gens originaires de diverses régions de l'ex-URSS et d'autres pays d'Europe de l'Est, viennent s'approvisionner et/ou vendre leurs marchandises : ils étalent leurs objets sur les trottoirs, près des gares, ou devant les magasins d'Etat fermés ou mal approvisionnés, ou encore occupent des stades entiers comme par exemple à Varsovie. Ces marchés ont généralement un label ethnique - d'après l'ethnicité majoritaire des vendeurs (marchés «russes» dans pratiquement chaque ville de Pologne, marché «polonais» comme celui de Berlin de 1989-92, marché "chinois» à Budapest). Les 
intermédiaires comme les agences de voyage qui gèrent le va-et-vient des touristes prolifèrent également. On peut distinguer quatre périodes dans l'évolution de ce phénomène des migrations marchandes post-communistes.${ }^{17}$. La première commence dès le début des années 80 pour s'intensifier après 89 et concerne surtout les Polonais, qui, n'ont pas attendu la chute du mur pour se mettre en route ${ }^{18}$. Ils ont été les premiers et les plus nombreux parmi les marchands ambulants et les travailleurs pendulaires de l'Europe de l'Est et ceci jusqu'à une date récente. Les formalités de voyage étaient assez souples en Pologne, comparativement à celles des autres pays de l'Est. La réglementation concernant les passeports de 1959, fut assouplie en particulier après le 1er janvier 1989. Les réglementations douanières facilitaient elles aussi la mobilité et les voyages d'affaires. La Pologne étant traditionnellement un pays d'émigration, la diaspora polonaise compte quelque 13 millions de personnes dans le monde et ses réseaux importants assurent l'accueil des nouveaux migrants et/ou facilitent leur passage. L'Allemagne était la destination la plus recherchée par les Polonais, non seulement à cause da sa proximité et une tradition migratoire vers ce pays, mais aussi parce que les barrières à l'entrée étaient réduites. Environ 1 million de ressortissants polonais ont été admis soit comme réfugiés politiques, soit comme Aussiedler (Allemands ethniques) dans les années 80 ; le visa allemand était facile à obtenir ou, dans certains cas, n'était même pas nécessaire (comme, par exemple, pour les séjours de moins d'un mois à Berlin-Ouest). Les premiers touristes polonais ayant voyagé en Occident étaient des vendeurs et acheteurs à la sauvette. Pour se procurer des devises étrangères (des DM essentiellement), ils ont commencé par vendre des marchandises apportées de Pologne, de RDA ou d'URSS. Cette vente aux frontières ou dans des lieux publics des grandes villes proches comme Vienne, Berlin, Budapest, transformait ceux-ci en "marchés polonais ", au fur et à mesure de l'occupation de l'espace par les marchands ambulants et autres. Ils vendaient tout ce qu'ils pouvaient transporter dans un grand sac de voyage ou dans une automobile. Avant même la chute du mur en novembre 1989, "le marché polonais » de Berlin était devenu un lieu de rencontre entre l'Est et l'Ouest; il annonçait l'ouverture prochaine. Le lieu a constitué pendant plusieurs années l'une des plus grandes attractions de la ville. A l'époque où Berlin était encore divisée en deux par le mur, les Polonais ont été les premiers à redécouvrir le vieux centre déserté autour de la Potsdamer Platz et à lui redonner vie. Ce n'était pas encore celui de tous les Berlinois, mais leur propre centre, entre Kreuzberg, où ils vendaient, la Bahnhof Zoo, où ils faisaient du change, et le mur près de Check-Point-Charlie, leur point de passage vers Berlin-Est où ils effectuaient leurs achats et prenaient trains et avions pour la Pologne. En 1990, 1991 et 1992, entre 10.000 et 100.000 Polonais venaient à Berlin chaque fin de semaine, et parfois même les autres jours. Pour eux le marché polonais de Berlin fut pendant quelques années ce pourvoyeur de l'égalité des chances, (equal opportunity employer), dont parlent Kornblum et Williams dans un autre contexte ${ }^{19}$. Pour quelques bibelots, une nappe brodée, un paquet de cigarettes, des lacets à dix centimes la paire, des jouets ou une bouteille de vodka, le vendeur polonais, qu'il fût enseignant, infirmier, ingénieur ou ouvrier, pouvait obtenir l'équivalent de son salaire mensuel et même plus. De 1988 à 1994 le nombre de voyages à l'étranger est passé de 8 millions, dont 1,7 seulement à l'Ouest, à plus de 30 millions par an. Soulignons que le nombre de personnes effectuant ces voyages était nettement inférieur, une personne pouvant effectuer plusieurs déplacements dans la même année; il est cependant impossible d'en connaître le chiffre précis, il faut se contenter d'estimations ${ }^{20}$. Quant à l'ensemble des passages aux 
frontières, toutes nationalités confondues, les gardes-frontières polonais en ont enregistré 262 millions en 1996. En même temps, alors que les échanges officiels entre les pays socialistes commençaient à se désintégrer, les arrangements informels se mettaient en place pour compenser les pénuries de certains produits de consommation courante. On enregistrait alors l'afflux de touristes venant d'Ukraine, de Russie et de Biélorussie en Pologne. Ils vendaient des produits des stocks d'entreprises de leurs pays aux Polonais qui allaient à leur tour les vendre à l'Ouest, et achetaient les produits polonais ou ceux que les Polonais ramenaient de l'Ouest. A la suite de ces mouvements qui continuent aujourd'hui, une nouvelle vague s'amorçait, constituée de marchands ambulants de l'ex-URSS qui se dirigeaient vers la Tchécoslovaquie, la Hongrie et la Yougoslavie pour acheter des produits de meilleure qualité, ils vendaient de l'or, des objets d'antiquités, des pièces détachées pour les voitures qui circulaient dans ces pays mais dont l'approvisionnement n'était plus régulier. Ces voyages étaient dans leur majorité individuels, sans organisation particulière. La troisième vague a commencé en 1991 et continue aujourd'hui. Les réseaux s'étendent à la Turquie, la Chine, les Emirats Arabes Unis, l'Inde. Les agences de tourisme se multiplient partout en ex-URSS et offrent des services (passeports, visas, transport). La publicité apparaît dans les médias montrant les marchandises qu'on peut acheter dans différents pays. Enfin, les années 1992/93 marque un tournant. Certains pays occidentaux, mais des pays comme la Pologne, la Hongrie, la Slovaquie et la République tchèque introduisent des restrictions. La distribution se privatise, les commerçants locaux voient donc d'un mauvais œil cette concurrence perçue comme déloyale. Les marchands ambulants ne s'installent plus n'importe où, ils disparaissent des rues, deviennent moins visibles, concentrés dans certains endroits (les stades), contrôlés par l'inspection et soumis aux taxations. L'Etat s'est mis à contrôler l'économie de bazar. Les tendances des flux se sont inversées en Pologne. Il y a désormais plus d'étrangers (notamment de l'ex-URSS, de Roumanie, de Bulgarie) qui viennent en Pologne que de Polonais qui en partent. Le nombre de voyageurs étrangers a rapidement augmenté, passant de 3,4 millions en 1985 à 61 millions en 1993. Les entrées de voyageurs de l'ex-URSS se sont dernièrement stabilisées à 8 millions, dix fois plus qu'il y a une dizaine d'années ${ }^{21}$. Comme les Polonais en Allemagne, les ressortissants de l'ex-URSS en Pologne reproduisent le modèle pendulaire des Polonais. Ils essaient d'améliorer leur niveau de vie chez eux, sans rompre leurs liens familiaux et culturels. S'installer en Pologne n'est pas attrayant, car cela impliquerait une baisse du statut social et économique, et s'installer à l'Ouest n'est pas réaliste. D'après un fonctionnaire de douane polonais: "Tant que les Russes peuvent faire du business en Pologne, ils ne seront pas intéressés par une éventuelle migration plus loin à l'Ouest ». Outre le commerce, ces voyages permettaient l'exercice de diverses autres activités, notamment dans les services (ménage, réparation de véhicules), dans l'agriculture ou dans le bâtiment. "La mobilité incessante des travailleurs polonais dans le secteur du bâtiment est aujourd'hui le simple effet de la demande, qui une fois satisfaite, replace le travailleur pendulaire dans sa mobilité antérieure " ${ }^{22}$. Il s'agit aujourd'hui, comme il y a un siècle, de maintenir la maind'œuvre comme une réserve mobilisable, mobile et disponible. Les questions concernant les droits politiques, la scolarisation, le logement, le débat sur l'intégration, etc., n'entrent pas en ligne de compte car le migrant n'évolue que dans la sphère pure du marché du travail, au sens plein du mot marché, c'est à dire comme lieu d'équilibre entre l'offre et la demande. De toutes les catégories de la migration de travail entre la Pologne et l'Allemagne, par exemple, la migration pendulaire est la plus importante en 
volume. On estime qu'il y a à tout moment environ 40.000 travailleurs polonais qui font la navette pour travailler sur les chantiers berlinois ${ }^{23}$. Les femmes travaillent pour la plupart dans les métiers de services où elles arrivent à gérer leur mobilité ${ }^{24}$, en fonction des obligations de travail ou de famille en Pologne. Mais aussi dans la prostitution, où leur mobilité est gérée par des gangs organisés spécialisés dans le trafic de femmes (qui apparemment rapporte plus que la drogue). On a estimé récemment à un demi-million le nombre de femmes prostituées en provenance des PECO, qui travaillent en Europe occidentale ; leur nombre serait environ 3000, dans la seule ville de Berlin ${ }^{25}$.

3 Les stratégies des pendulaires: la mobilité comme ressource Les migrants circulant pour faire du commerce ou pour travailler sont au cœur des transformations qui marquent les PECO après la chute du système communiste. On voit resurgir une économie parallèle fondée sur la mobilité, sur des solidarités précaires et changeantes, dont les acteurs sont ces femmes et ces hommes toujours prêts à partir, indifférents aux frontières et aux distances. Ils s'accommodent d'une vie temporairement éclatée entre leur «chez soi » et leur itinérance qui remplit une bonne partie de leur vie. Ils conservent cette disponibilité à la mobilité voire à la réinstallation ailleurs, car elle est actuellement la seule garante de la survie pour la majorité d'entre eux, d'une meilleure qualité de vie et, pour un petit nombre elle assure l'accumulation du capital. Comment expliquer les stratégies de ces migrants circulant dans l'espace transnational construit au fil de leur itinérance? Nous essayons d'appliquer au phénomène de migration pendulaire marchande et de travail l'approche développée dans la recherche sur l'ethnic business. La "mise en route» peut-elle être analysée comme la mise à son compte ? La stratégie économique du migrant pendulaire est-elle similaire à la stratégie d'un entrepreneur ethnique ? On sait, à partir des travaux sur le ethnic business ${ }^{26}$ que pour certains immigrés, l'entrepreunariat est la réponse à la mobilité sociale bloquée suite à des restructurations dans des industries dans lesquelles ils étaient recrutés comme main-d'œuvre bon marché et peu ou pas qualifiée ; leur avancement ou même leur maintien comme travailleur salarié étant compromis compte tenu de leur profil, ils se mettent à leur compte pour faire face aux obstacles qu'ils rencontrent sur le marché du travail. Ils ont recours aux réseaux de solidarité communautaires et aux ressources spécifiquement "ethniques». Ainsi une "opportunité bloquée » peut se trouver à l'origine de nouvelles opportunités. Le modèle théorique interactif de l'ethnic business est construit à partir de deux dimensions : structures d'opportunité et caractéristiques communautaires des migrants. Les stratégies entrepreneuriales des migrants sont le résultat de l'interaction ou de la fusion entre la «structure et la culture " ${ }^{27}$. Bloqués à l'entrée dans le "mainstream capitalist economy », les immigrants créent leur propre "capitalism» ${ }^{28}$. Les entrepreneurs ethniques s'adaptent aux contraintes de la structure sociale (conditions du marché, politiques publiques) et en s'appuyant sur leurs caractéristiques - "prédispositions », expérience passée, des réseaux, ressources communautaires - ils essaient de se tailler leur propre "niche» dans le marché. Ils parviennent ainsi à transformer ce qui a initialement été un handicap en un avantage. Nos précédentes recherches sur le secteur de l'habillement et lors d'une recherche sur les femmes immigrées créatrices d'entreprises, s'appuyant sur ce modèle nous a permis d'établir que le recours à la solidarité collective n'était pas limité à la communauté d'origine et impliquait souvent une solidarité trans-communautaire des femmes. Ceci semble être le cas aussi dans les migrations dans l'Est Européen, où on observe également le recours à des solidarités plus étendues, au-delà des groupes communautaires. On peut distinguer deux situations types (dont la seconde, surtout, 
nous intéresse). La première, concerne l'entrepreneur « ethnique » classique, installé, qui profite de la mobilité des autres. Il a recours à la main-d'œuvre bon marché et surtout mobile, de ses compatriotes ; la mobilité de ses travailleurs permet de masquer l'embauche au noir. Il faut y ajouter toute sorte d'intermédiaires (logeurs, agences matrimoniales, proxénètes, passeurs) qui font fonctionner l'économie informelle au sein de laquelle l'insécurité et la mobilité des migrants sont des atouts importants et des sources de revenus. Ces intermédiaires cherchent à élargir leur champ d'action audelà de la communauté de compatriotes stricto sensu. La traite de femmes dans divers pays de l'Est par des réseaux organisés, s'appuie sur un système de rotation entre différentes capitales européennes: le nomadisme sexuel qui est ainsi imposé à ces femmes et jeunes filles est rythmé au gré de leur visas ou autorisations de séjour touristique, et met les gangs à l'abri du contrôle policier et des poursuites judiciaires. La seconde situation, et de loin la plus fréquente, concerne les migrants qui gèrent leur propre mobilité en fonction de différentes contraintes du marché. Pour eux la migration n'est qu'une alternative attrayante à l'émigration: elle leur épargne l'investissement psychologique et financier, ainsi que le choc culturel qu'impliquerait une tentative d'installation dans un autre pays, ne serait-ce que pour une durée limitée. Les personnes qui se sont mises en route ont comme objectif d'essayer d'améliorer, voire simplement de maintenir leur niveau de vie chez eux. Pour ce faire, la majorité d'entre elles mettent en relation les deux systèmes, celui de départ et celui d'arrivée, et construisent un champ migratoire propre, dans lequel elles optimisent leur capacité de faire face aux obstacles, mais aussi de gérer les opportunités et les défis émanant des deux systèmes. Ce faisant elles choisissent $d^{\prime}$ '« ignorer » certaines règles en vigueur à la fois dans leur pays d'origine et dans celui (ou ceux) qu'elles traversent, où elles travaillent ou font du commerce : elles sont souvent en situation irrégulière ou leur activité frôle l'illégalité. Comment se fait la rencontre entre «structures d'opportunité » et "caractéristiques de groupe » chez ces deux types de migrants de l'Est? Que dire des stratégies adoptées par des pendulaires de l'Est? En fait, ces gens ont ajusté leur comportement migratoire en réponse à des changements survenus à la fois dans les économies en transition (de leur pays et d'autres pays de la région) et à l'évolution de la situation dans les pays de destination (notamment des politiques migratoires de pays de l'Ouest ou de ceux l'Europe centrale).

Structures d'opportunité Avant 1989, l'immigration, en Allemagne surtout, a été largement facilitée pour les Allemands ethniques originaires de Pologne, de l'URSS et de Roumanie, grâce à la souplesse des mesures concernant l'accès au pays et sur le marché du travail. Les demandeurs d'asile politique en provenance du bloc communiste étaient également accueillis à bras ouverts et constituaient une catégorie de réfugiés privilégiés (ils avaient l'accès au marché du travail). Par conséquent, au moment de la chute du mur une population stable de naturalisés ou de résidents privilégiés était présente, prête à accueillir des populations qui allaient se lancer sur les routes et leur servir de relais. Après 1989 de nombreuses mesures prises par les pays de l'Union européenne ont fortement facilité la mobilité des habitants de cette région. Par ailleurs, certaines restrictions prises progressivement à l'Ouest et plus tard par certains pays de l'Europe Centrale ont encore contribué à cette mobilité. Sur le plan politique il existe des différences notables entre les réglementations des pays aussi bien quant à leur nature qu'à leur rythme d'application. Néanmoins, dans l'espace de l'Union européenne, ce sont l'abolition des visas de sortie, la levée des restrictions pour l'obtention de passeports, l'abolition des visas pour les citoyens européens et certains 
citoyens non-européens, la ratification de la Convention de Genève, les accords sur les recrutements de main-d'œuvre à l'Ouest, qui constituent les dispositions les plus importantes. En Occident, des mesures plus restrictives ont été introduites notamment en ce qui concerne la demande d'asile et la reconnaissance du statut d'Allemand ethnique ; l'accès au marché du travail a été également de plus en plus réglementé. Ces mesures, tout en contribuant à la mobilité transfrontalière, ont diminué la possibilité d'installation durable. La mobilité a été encore facilitée par la proximité géographique et le faible coût du transport qui réduisait encore plus les distances. Enfin, et ceci est tout particulièrement caractéristique des pays de l'Europe centrale, grâce à une certaine combinaison de facteurs, tels que la sécurité, le respect d'un minimum de règles dans l'espace public et la tolérance ou le laisser-faire envers certaines irrégularités (comme par exemple, les marchés informels, ou l'emploi au noir), ces pays inspirent confiance et rendent cette zone attrayante pour des investisseursentrepreneurs étrangers ou des marchands pendulaires. Mais il peut arriver que du jour au lendemain, des mesures restrictives ou des interdictions se substituent à la tolérance. Les migrants-commerçants plient alors leurs bagages. C'est le cas des Polonais qui ont quitté « leur marché » de Berlin, lorsqu'il ne fut plus toléré, des Russes qui ont cessé leurs navettes en Norvège et des Chinois qui ont quitté la Hongrie, lorsqu'une nouvelle législation fut adoptée (ou la législation appliquée), ce qui rendait leur commerce impossible ou peu rentable. Sur le plan économique, la transition à l'économie du marché dans l'Union européenne a révélé l'existence d'un surplus de main-d'œuvre sur place, ce qui a conduit à la diminution d'emploi et le chômage. La baisse de la production, le mauvais approvisionnement en articles même les plus élémentaires de la consommation courante, parallèlement à la présence et relative accessibilité de stocks de la production socialiste de masse, complètent ce spectre d'opportunités de part et d'autre des frontières. La mobilité facile permettait de connaître et d'apprécier soi-même les atouts et les inconvénients d'ici et de là-bas dans une perspective globale, donc d'envisager les projets autrement qu'en fonction de l'unique alternative "rester ici ou s'exiler là-bas" ${ }^{29}$. Parmi les caractéristiques de groupes les théoriciens de l'ethnic business distinguent, d'une part, les " prédispositions » (c'est-à-dire le capital social : expérience antérieure, la présélection par les conditions de migration, expérience de la «situation bloquée » sur le marché du travail) et de l'autre, la mobilisation de ressources (réseaux et ressources communautaires, le savoir-faire spécifique). Les réseaux des pendulaires sont transnationaux, tout en ayant un fondement ethno-culturel. Plus de la moitié des personnes interrogées lors de nos enquêtes ont de la «famille» ou des «amis » en Allemagne ou partout ailleurs sur leur parcours. Grâce à eux, il est plus aisé d'affronter les obstacles bureaucratiques, de trouver du travail ou un logement, les invitations, lettres de références ou de recommandation n'étant qu'un aspect de cette aide. Le passeport allemand de membres de la famille appartenant au réseau peut contribuer à "prouver» l'origine allemande lors d'une demande de naturalisation ${ }^{30}$. La force des liens ainsi établis ne provient pas de leur ancrage dans des groupes communautaires, mais au contraire de leur fonctionnalité et de leur efficacité quant aux objectifs poursuivis. C'est la fonction, l'activité qui compte et non l'existence des liens primaires. En ce sens, ces liens se rapprochent de ce que Marc Granovetter appelait les «weak ties " ${ }^{31}$. Ces liens mettent en relation les membres de groupes différents (contrairement aux «strong ties » qui assurent une cohésion intra-groupe et que l'on trouve souvent justement dans l'ethnic business). Qu'il s'agisse de planter des fraisiers 
dans le sol glacé en Suède ou en Norvège, de poser du carrelage à Hanovre, de se faire embaucher pour des récoltes dans le Palatinat, de faire du nettoyage industriel dans le port de Brême, de fabriquer des prothèses dentaires, de rénover ou nettoyer des appartements à Berlin ou de réparer des voitures, tous ces emplois précaires ont été trouvés grâce aux intermédiaires polonais, de vagues "cousins » ou "voisins » ou « amis » qui ont déjà eux-mêmes travaillé sur place ${ }^{32}$. D'autres cherchent à exploiter la confiance de leurs compatriotes et on peut difficilement parler de "solidarité communautaire ». Certaines entreprises du bâtiment créées par des Germano-Polonais (généralement dans une relation de sous-traitance vis-à-vis de grandes entreprises du bâtiment) en sont l'un des nombreux exemples. Dans ce secteur en crise, les grandes entreprises transfèrent leurs risques aux sous-traitants, tandis que ceux-ci cherchent à économiser sur les salaires et les conditions de travail de leurs ouvriers. Les contrats sont verbaux et l'inégalité dans le rapport de pouvoir entre l'employeur et ses ouvriers n'en est que plus accentuée. Les ouvriers se plaignent de ne recevoir que des salaires « russes » (c'est-à-dire d'un niveau plus bas qu'en Pologne) ou de n'être pas payés du tout. Il est en effet courant que l'employeur remette constamment à plus tard le versement des sommes dues, en profitant de la confiance et de la loyauté que lui témoigne initialement le travailleur. Avec le temps, les dettes envers ce dernier s'accumulent, tandis que lui-même ne parvient pas, parce qu'il attend le règlement de son dû, à se défaire d'un rapport où il est de plus en plus perdant ${ }^{33}$. Pour les pendulaires, qui font des haltes de courte durée pour commercer, le fonctionnement des réseaux est encore plus dicté par leur activité. Les réseaux se forment dans un territoire où les solidarités précaires fonctionnent le temps d'un voyage, pour se dissoudre aussitôt après et se reconstituer de nouveau avec d'autres personnes ou lors d'un nouveau trajet. Des liens ne se forment pas tant sur les bases ethniques, que sur les bases de l'expérience commune à ceux qui partagent la même route, investissent les mêmes espaces et ont à faire aux mêmes intermédiaires (agents de voyage ${ }^{34}$, guides, recruteurs, logeurs, garde-frontières, douaniers). Par ailleurs, la transition à l'économie de marché a produit des gens disponibles. Formellement ils ont un emploi et des droits sociaux hérités du socialisme, mais souvent ne reçoivent pas de salaire ou reçoivent des salaires insuffisants pour vivre. Ils ont peu d'obligations de présence dans ces conditions (ou ils s'arrangent entre collègues de travail pour établir une rotation de présence). Ils ont beaucoup de temps, leur temps est bon marché et ils sont prêts à le vendre à un prix très bas. Des Ukrainiens, des Biélorusses, des Bulgares, des Roumains sont dans cette situation. Leur mobilité les amène rarement jusqu'à l'Occident destination quasiment réservée aux Polonais, qui ont monopolisé des emplois saisonniers dans l'agriculture, dans le bâtiment ou les services rendus aux particuliers. La ressource spécifique, mais non ethnique, de ces migrants ou de ceux qui les emploient est donc cette mobilité incessante. Elle permet de vendre ou de se vendre à un autre endroit meilleur que chez soi. Parfois la mobilité est effectuée sans destination précise. Ce qui importe est le fait d'être en route ${ }^{35}$. La mobilité permet aussi d'éviter l'illégalité. On repart avant que le visa ou le séjour "touristique » expire. Lorsqu'ils sont à la recherche d'environnements politico-economiques plus cléments et plus propices pour faire des affaires, la disponibilité à la mobilité est un atout important. Il s'agit donc de gens qui, forts de leur expérience acquise à l'époque communiste lorsqu'il s'agissait de court-circuiter le système pour survivre, font appel à des solidarités familiales, professionnelles ou autres comme substituts lors de leurs absences plus ou moins prolongées de chez eux. Ils profitent des déséquilibres 
structuraux, des failles dans la législation et des imperfections du marché pour se mettre en route ou pour utiliser ceux et celles qui sont mobiles. Ils peuvent compter sur des réseaux de « compatriotes " (dont l'étendue est plus souvent panslave que limitée à une seule nationalité), intermédiaires spécialisés dans tel ou tel « service » de gestion de la mobilité (leur propre, ou celle des autres).

Stratégies L'entreprise ethnique des années 80 est en quelque sorte le fait du migrant installé, intégré, stable. "Celui qui cultive le projet de retour, ne prend pas de risque et reste en bas de l'échelle sociale. Par contre, celui qui veut s'installer durablement n'acceptera pas cette situation et cherchera la promotion sociale en prenant des risques et en se mettant à son compte " ${ }^{36}$. Les enquêtes portent essentiellement sur les immigrés ayant résidence permanente dans le pays où ils se consacrent au business. Le business suppose donc une certaine stabilité. La mobilité est d'abord impossible. Elle apparaît cependant plus tard, comme le résultat du fonctionnement, de l'extension ou de la diversification de l'activité entrepreneuriale. Des recherches plus récentes attirent toutefois l'attention sur les migrants entrepreneurs, ouverts à la mobilité et prêts à explorer les possibilités dans un troisième, voire quatrième pays. Les migrants que nous avons observés ne cherchent pas à s'installer. S'ils ont un projet d'établissement à leur compte, c'est chez eux ou dans des marchés plus officiels de la zone frontalière. Qu'il s'agisse de commerce ou de travail, une partie considérable de leur vie est consacrée à une activité qui repose sur une mobilité incessante : plusieurs années et, pour certains, dix, ou même quinze ans. Là où nous parlons de migration, immigration ou intégration, ces personnes ne parlent que de « voyages ». La question, par exemple, du retour n'a souvent aucun sens pour ceux qui sont en route mais ne se considèrent pas comme étant partis. Pendant cette période de mobilité, leur activité peut se transformer plusieurs fois, passant du commerce au travail au noir, du trafic sur le change au travail saisonnier dans l'agriculture, ou de nouveau au commerce. Par ailleurs, contrairement à la majorité des entrepreneurs ethniques ou immigrés en France, aux Etats Unis ou ailleurs qui se consacrent entièrement à leur activité, souvent 10 à 12 heures par jour et sept jours par semaine, les migrants pendulaires de l'Est quelle que soit leur activité, et surtout lorsqu'ils sont libres dans la gestion de leur mobilité, cherchent à préserver leur travail chez eux et en même temps à préserver leurs liens familiaux. Ils vivent réellement entre deux mondes ${ }^{37}$. Ainsi développent-ils ce que nous avons appelé la "rotation autogérée " ${ }^{38}$. Les travaux saisonniers dans l'agriculture sont, par exemple, facilement compatibles avec les horaires d'une personne travaillant dans l'enseignement, et ceux qui travaillent dans le bâtiment ou font de la réparation de voitures, peuvent le faire grâce aux substituts pour leur travail chez eux. Les femmes, pour la plupart, travaillent dans le secteur des services aux particuliers, comme femmes de ménage, aides-soignantes ou serveuses. Cette rotation autogérée peut prendre l'allure d'un véritable système de partage de l'emploi : par exemple, certaines femmes, généralement pas plus d'un groupe de cinq, alternent des périodes de travail en Allemagne avec d'autres, plus longues, en Pologne. Ainsi, tout en travaillant chez des employeurs allemands, elles restent disponibles et présentes dans leurs familles, quoique plus rarement pour leur travail en Pologne. La rotation est donc dictée par les obligations familiales en Pologne ${ }^{39}$.

6 Conclusion

7 Les migrants pendulaires de l'est européen, urbanisés, d'un niveau de scolarisation et de qualification relativement élevé, remettent en question un certain nombre d'idées 
reçues, héritées de la vision dichotomique et statique du phénomène migratoire (qui suppose l'exclusivité des allégeances) : la migration ne serait que le passage obligé entre deux sédentarités, en somme, l'outil du sédentaire; la mobilité sociale ascendante, la réussite, dépendraient de l'installation dans le pays d'arrivée, alors que le maintien de contacts et l'orientation vers le pays d'origine y feraient obstacle. A cette vision de la «migration rupture" l'itinérance des pendulaires s'oppose comme «migration maintien", pour reprendre la distinction que propose Rozental dans un contexte de migrations internes. Les pendulaires montrent qu'ils ne sont pas seulement les victimes de l'économie en transition. Leur migration ne se réduit pas à une stratégie de survie, mais devient aussi recherche de sens, d'indépendance et de promotion sociale. Les écarts de richesse entre leur pays et ceux de leur parcours, pays désormais à portée de main, sont des opportunités à saisir, auxquelles ils répondent en mobilisant la ressource qui leur procure un avantage de taille sur celui qui ne bouge pas à savoir, non seulement la mobilité, mais aussi la capacité de rester mobile pendant longtemps. La phase de circulation ou la migration pendulaire peut être particulièrement longue. Après une longue période d'installation dans la mobilité, les migrants ne cherchent qu'à rentrer chez eux. La migration aurait alors un sens contraire à celui qui lui a été le plus souvent attribué : elle serait une stratégie pour ne pas partir, une alternative à l'émigration. D'ailleurs, en ce qui concerne l'Europe occidentale, compte tenu de l'absence d'une politique d'immigration, les pendulaires n'ont guère d'autre possibilité que de circuler. La transformation de cette migration temporaire en migration permanente, à l'instar de ce qui s'est produit en partie avec la migration " temporaire " des années 60-70, est peu probable. Le paradis n'est plus au-delà des frontières mais est recherché en deçà, pourvu que l'on sache rentabiliser sa disponibilité à la mobilité. Outre l'absence d'obstacles à la circulation (proximité, faible coût de transport, ouverture des frontières), les recherches sur le terrain ont permis de distinguer les facteurs qui rendent cette transformation encore plus difficile. Le déclassement par rapport à la situation au pays (cas très fréquent parmi ceux que nous avons observés), joue comme repoussoir pour les migrants et ceux-ci cherchent à limiter d'eux-mêmes la durée de leur séjour et à organiser leur travail à l'étranger de manière intermittente. La durée de séjour joue aussi. Moins les séjours seront prolongés, moins les migrants auront l'occasion de créer de nouveaux liens, plus durables, et de transformer leurs projets d'origine. En cela, ils sont différents des « Gastarbeiter » des années 60-70. Ils ne perçoivent pas leur séjour à un horizon de quelques années, mais de quelques semaines ou mois, voire de quelques jours. L'existence d'une alternative à l'émigration diminue le risque d'une émigration de longue durée. Ainsi plus les options des gens sont multiples et variées, moins ils seront tenus à prendre des décisions sous pression et sous forme définitive et exclusive. L'existence des réseaux et des attaches familiales au pays est le facteur prépondérant. Le pendulaire en fait n'est jamais vraiment parti de chez lui. Il ne s'agit donc pas de nouvelles vagues de travailleurs « qui ont l'intention de retourner un jour et qui finiront par s'installer ", mais des personnes effectivement temporaires et ceci non seulement d'après leurs intentions. Chez les migrants circulant à l'intérieur de l'espace des PECO, l'orientation vers l'Occident comme destination est absente ${ }^{40}$. Comme les écarts de richesses entre les pays de l'Union européenne et les pays plus à l'Est et au Sud augmente, la migration-circulation va continuer encore longtemps. 


\section{NOTES}

1. Cet article reprend une communication présentée dans le cadre du colloque «Réseaux transnationaux entre l'Europe et le Maghreb », Université d'Aix en Provence - IREMAM, 29 juin au 1er juillet 1998.

2. Voir l'analyse comparative France-Allemagne in Morokvasic M., Angenendt S., Fischer A. (eds.), « Migrations est/ouest dans le débat scientifique et politique en France et en Allemagne ", Revue d'études comparatives est-ouest, n³, 1994, pp. 135-160.

3. Certains titres de cette littérature sont à ce propos significatifs : One way Ticket, Ils resteront ici, Here for good.

4. Pour éviter toute confusion avec la libre circulation au sein de l'Union européenne, signalons que la libre circulation dont bénéficient les ressortissants est-européens n'implique pas le droit au travail et à l'installation.

5. D'après les enquêtes de terrain en Pologne, l'augmentation du volume de migrations après 1989 est plus attribuable à la multiplication de la circulation des mêmes personnes, qu'à l'augmentation du nombre des personnes qui circulentJazwiska Ewa, Okolski Marek, Causes and Consequences of migration in Central and Eastern Europe, Institute of Social Studies, University of Warsaw, 1996.

6. Cf. SOPEMI, Tendances des migrations internationales, Paris, OCDE, 1995 ; Salt John, Current and future trends in international migration in Europe, Strasbourg, Conseil de l'Europe, CDMG (95) 6E., 1996 ; Simon Gildas, Géodynamique des migrations internationales dans le monde, Paris, PUF, 1995 ; de Tinguy Anne, Wihtol de Wenden Catherine (dir.), L'Europe et toutes ses migrations, Bruxelles-Paris, Complexe, 1995 ; Okolski Marek, « New migration trends in Central and Eastern Europe in the nineties. How significant, how stable? », communication à l'International Migration at Century's End, IUSSP Committee on South North Migration, Barcelona, 7-10 mai 1997;

Morokvasic M., Angenendt S., Fischer A. (eds.), op. cit. ; Morokvasic Mirjana, Rudolph Hedwig (dir.), Migrants. Les nouvelles mobilités en Europe, Paris, L'Harmattan, 1996, pp.119-157 ; Wallace Claire et al., Spending, Saving or Investing Social Capital : the Case of Shuttle Traders in Post-Communist Central Europe. East European Series, $\mathrm{n}^{\circ} 43$, Institute for Advanced Studies. Vienna, 1997.

7. Tarrius Alain, Les fourmis d'Europe. Migrants riches, migrants pauvres et nouvelles villes internationales, Paris, Harmattan, 1992 ; Peraldi Michel, « Portraits d'entrepreneurs ", in Ces quartiers dont on parle (collectif), La tour d'Aigues, Ed. de l'Aube, 1997.

8. Cf. Césari, Jocelyne, «Transnational networks between Europe and North Africa. A risk for the nation-states? ", communication au XVII World Congress of the International Political Science Association, 17-21.8.1997, Seoul, Corée, 1997.

9. Voir entre autres Glick Schiller Nina, Basch L., Blanc-Szanton C. (eds.), Towards a Transnational Perspective on Migration, New York, New York Academy of Sciences, 1992.

10. Cf. Morokvasic Mirjana, Rudolph Hedwig, Wanderungsraum Europa. Menschen und Grenzen in Bewegung, Berlin, Sigma, 1994. 
11. Schierup Carl-Ulrik, «Eurobalkanism. Ethnic cleansing and the post cold war order ", communication présentée à la Conférence internationale : The Yugoslav War and Security in the Balkans and in Europe, Bologna, 10-11 décembre 1993.

12. Widgren Jonas, « Migration als Politicum » in Morokvasic Mirjana, Rudolph Hedwig, Wanderungsraum Europa..., op. cit., pp. 74-80 ; Morokvasic Mirjana, Rudolph Hedwig (dir.), Migrants. Les nouvelles mobilités..., op. cit. ; cf. également l'observatoire International des Migrations, divers documents sur la migration de transit, Genève, IOM-Migration Information Programme, 1995.

13. En volume c'est le couple Allemagne-Pologne qui est le premier concerné, 300.000 Polonais par an étant recrutés dans le cadre de divers programmes. Les Polonais se dirigent aussi vers d'autres pays, les Tchèques vont travailler en Autriche et en Allemagne, les Albanais en Italie et en Grèce, les Russes et les Estoniens en Finlande ; la Pologne, la Hongrie et la République tchèque reçoivent des travailleurs de Bielorussie, de Roumanie et de Russie, etc.

14. Einhorn Barbara, Cinderella goes to market. Citizenship, Gender and Women's Movements in East Central Europe, London, Verso, 1993.

15. Domernik Jeroen, Going West. Soviet-Jewish Immigrants in Berlin since 1990, Aldershot, Avebury, 1997.

16. Voir différentes études de l'Organisation Internationale des Migrations, Transit Migration in Poland, Genève, IOM-Migration Information Programme, 1994.

17. Wallace Claire et al., op. cit.

18. Des Polonais m'ont raconté cette blague à leur propos : « être anglais est une nationalité, être français aussi, être polonais c'est une profession ».

19. Kornblum William, Williams T., Growing up Poor, Lexington, MA, Lexington Books, 1985.

20. Ainsi en 1989 et en 1990, plus de 2,5 millions de Polonais ont obtenu le visa allemand ; avec un visa, ils effectuaient entre cinq et dix voyages, selon nos observations à la frontière, cf. Morokvasic M., « Une migration pendulaire : les Polonais en Allemagne », Hommes et Migrations, $n^{\circ} 1155,1992$, pp. 31-37.

21. Cf. l'Organisation Internationale des Migrations, Transit Migration in Poland, op. cit., 1994.

22. Cf. Weber Frank Paul, « Des migrations du travail en Allemagne : le territoire introuvable » in Morokvasic Mirjana Rudolph Hedwig (dir.), op. cit., pp.159-184, 1996. 23. Cf. Cyrus Norbert, Den Einwanderungskontrollen entgangen.Bestandsaufnahme und Anmerkungen zur unkontrollierten Zuwanderung in die Bundesrepublik Deutschland am Beispiel polnischer Staatsangehöriger, Polnischer Sozialrat, Berlin, 1997.

24. La « rotation autogérée ", cf. Morokvasic Mirjana et Rudolph Hedwig (dir.), op. cit. 25. Cf. Cyrus Norbert, op. cit. ; Le Monde, 26-27 avril 1998.

26. Notamment ceux de Waldinger Roger et al, Ethnic Entrepreneurs, London, Sage, 1990 ; de Light Ivan, Bonacich Edna, Immigrant Entrepreneurs. Koreans in Los Angeles, Berkeley, Los Angeles, University of California Press, 1988 ; ainsi que de nombreux travaux en France.

27. Cf. Waldinger Roger et al, op. cit.

28. Portes Alejandro, « Modes of Structural INcorporation and Present Theories of Labor Immigration », in Kritz Mary, Keely B. Charles, Tomasi M Silvano, Global Trends in Migration, New York, Centre for Migration Studies, 1981. 
29. Morokvasic Mirjana, «Les élites scientifiques en provenance de l'Europe de l'Est : exode ou circulation », Revue d'études comparatives est-ouest, n³ 3, 1996, pp.31-73 ; « Entre l'Est et l'Ouest... », op. cit.

30. Notons cependant que la naturalisation allemande ne signifie pas automatiquement l'installation durable et définitive sur le territoire allemand. Elle ne fait que faciliter, ou, si l'on veut, normaliser un mode de vie qui continue comme avant dans un espace germano-polonais, avec beaucoup de va-et-vient.

31. "The strength of weak ties », American Journal of Sociology, 1971.

32. Morokvasic Mirjana, « Entre l'Est et l'Ouest... », op. cit.

33. Il peut arriver que l'employeur « disparaisse » ou que le travailleur se fasse prendre lors d'un contrôle de police juste au moment où son dû allait lui être réglé. $\mathrm{Cf}$. Weber Frank Paul, op. cit.

34. A présent, ceci concerne moins les citoyens polonais qui bénéficient d'une relative libre circulation, que les ressortissants de l'ex-URSS. Sur le marché « russe » de Varsovie ou à Bialystock, un Russe, Biélorusse ou Ukrainien pouvait encore en décembre 1993 acheter pour 4.000 zlotys (1 DM environ) des vouchers (attestations des « agences de voyages » nécessaires pour l'obtention d'un visa). L'acheteur inscrivait luimême son nom.

35. Wallace, Claire et al., op. cit.

36. Waldinger Roger et al, op. cit.

37. Cf. Morokvasic Mirjana, " Une migration pendulaire... », op. cit. ; Morokvasic Mirjana, « Entre l'Est et l'Ouest : des migrations pendulaires » in Morokvasic Mirjana, Rudolph Hedwig (dir.), op. cit.

38. Cf. Simon Gildas, op. cit., sur les retours alternatifs.

39. Morokvasic Mirjana, « Entre l'Est et l'Ouest... », op. cit.

40. Wallace Claire et al., op. cit.

\section{INDEX}

Index géographique : Europe centrale et orientale

\section{AUTEUR}

\section{MIRJANA MOROKVASIC-MULLER}

Chargée de recherches au Laboratoire d'Analyse des Systèmes Politiques, CNRS, Université ParisX-Nanterre. 